Review article

\title{
Right posterior segment graft for living donor liver transplantation: A systematic review
}

\author{
Kosei Takagi ${ }^{\text {a,b,*, Piotr Domagala }}{ }^{\mathrm{a}, \mathrm{c}}$, Wojciech G. Polak ${ }^{\mathrm{a}}$, Jan N.M. Ijzermans ${ }^{\mathrm{a}}$, Markus U. Boehnert ${ }^{\mathrm{a}}$ \\ a Department of Surgery, Division of HPB E Transplant Surgery, Erasmus MC, University Medical Centre Rotterdam, Dr. Molewaterplein 40, 3015, GD, Rotterdam, The Netherlands \\ b Department of Gastroenterological Surgery, Okayama University Graduate School of Medicine, Dentistry, and Pharmaceutical Sciences, 2-5-1 Shikatacho, Kita-ku, Okayama, Japan \\ c Department of General and Transplantation Surgery, The Medical University of Warsaw, Nowogrodzka 59 St., 02-006 Warsaw, Poland
}

\section{A R T I C L E I N F O}

\section{Keywords:}

Right posterior segment graft

Living liver transplantation

Outcome

\begin{abstract}
A B S T R A C T
The clinical significance of the right posterior segment (RPS) graft in living donor liver transplantation (LDLT) is unknown because of its limited use and technical concerns. This study aimed to review published studies investigating outcomes of RPS grafts. The systematic literature search was conducted to retrieve data from Embase, Medline Ovid, Web of Science, Cochrane CENTRAL, and Google Scholar. Among the 388 articles, six retrospective studies from Asian countries were included. The overall incidences of major and minor complications after RPS graft procurement were $5.6 \%$ and $34.6 \%$, respectively and no donor deaths were reported. RPS graft recipients had the following postoperative complications: overall mortality rate, $14.5 \%$; bile leakage, $8.7 \%$, biliary stenosis, $18.8 \%$, hepatic artery thrombosis, $8.7 \%$, and liver re-transplantation, $2.9 \%$. The RPS graft can be considered as an option for a living liver graft respecting donor safety under strict selection criteria and surgical strategy. The precise evaluation and understanding of anatomical variations and volumetric analyses is critical for selecting donors and planning the surgical strategy in the RPS grafts procurement. The RPS grafts procurement requires carefully dissection of the hepatic artery and portal vein, safely confirmation of the bile duct, and precisely parenchymal transection. However, further experience is needed to clarify the significance of the RPS graft in LDLT. The special technical requirements should limit this donor procedure to centers with a high level of experience in LDLT.
\end{abstract}

(c) 2019 Elsevier Inc. All rights reserved.

\section{Contents}

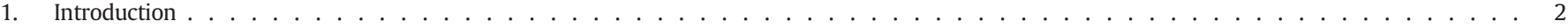

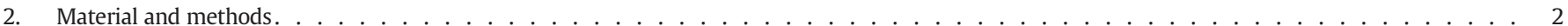

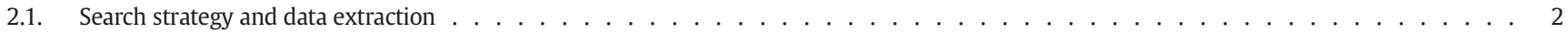

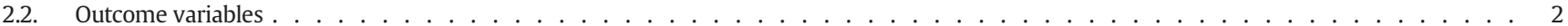

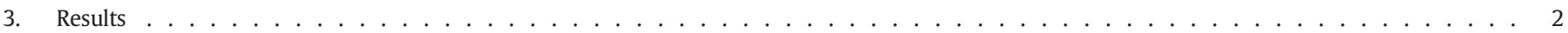

3.1. Characteristics of included studies . . . . . . . . . . . . . . . . . . . . . . . . . . . . . . 2

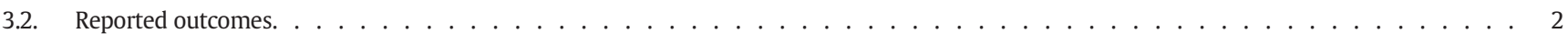

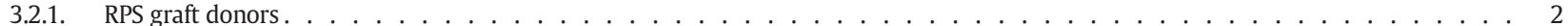

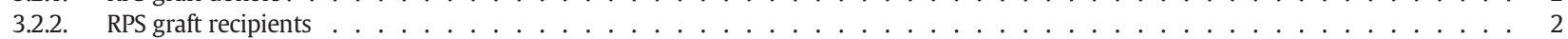

3.2.3. Donor selection for the RPS graft . . . . . . . . . . . . . . . . . . . . . . . . . . . . 3

3.2.4. Anatomical challenges of the RPS graft procurement $\ldots \ldots \ldots \ldots$

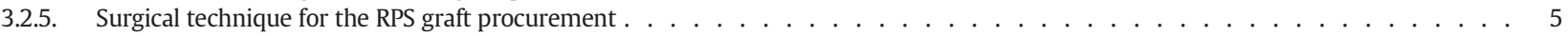

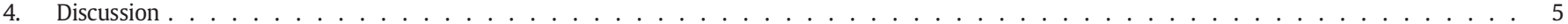

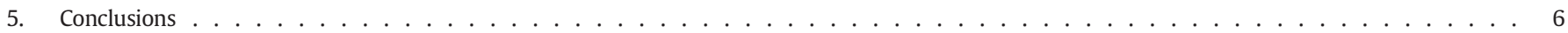

Abbreviations: CDc, Clavien-Dindo classification; 95\%CI, 95\% confidence interval; GRWR, graft-to-recipient weight ratio; HA, hepatic artery; HAT, hepatic artery thrombosis; HD, hepatic duct; LDLT, living donor liver transplantation; MELD, Model for End-Stage Liver Disease; PV, portal vein; SLV, standard liver volume; RHV, right hepatic vein; RL, right lobe; RPS, right posterior segment; RR, risk ratio.

* Corresponding author at: Department of Surgery, Division of HPB and Transplant Surgery, Erasmus MC, University Medical Centre Rotterdam, Dr. Molewaterplein 40, 3015, GD, Rotterdam, The Netherlands.

E-mail addresses: kotakagi15@gmail.com (K. Takagi),w.polak@erasmusmc.nl (W.G. Polak), j.ijzermans@erasmusmc.nl (J.N.M. Ijzermans). 


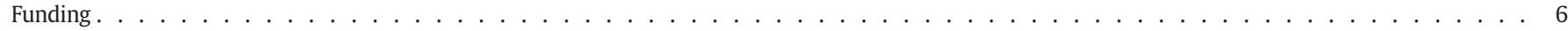

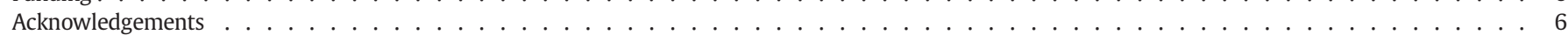

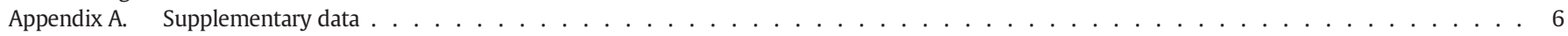

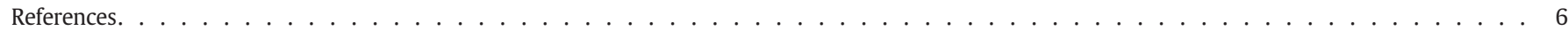

\section{Introduction}

Liver transplantation has become an established treatment for patients with end-stage liver diseases. In Western countries, the majority of liver transplantation is deceased donor liver transplantation. Therefore, living donor liver transplantation (LDLT) is recognized as an alternative to increase the donor pool and reduce the waiting list mortality of patients [1,2]. In many Asian countries, LDLT accounts for the majority of liver transplant procedures because of the severe deceased organ shortage, which has led to the development of unique technical and logistical innovations in LDLT [3]. However, LDLT is still under a high level of scrutiny because of ethical issues including the risk of morbidity and mortality in donors [4].

The right lobe (RL) graft and the left lobe (LL) graft were the majority of grafts used in adult-to-adult LDLT. However, we often encounter the problem within limited graft selection, particularly in donors with consecutive graft-volume (GRWR - Graft Recipient Weight Ratio) mismatch. Insufficient volume of the left lobe (LL) graft would lead to small-for-size graft syndrome for recipients. Donation of the right lobe (RL) graft is limited by the minimal liver remnant in order to avoid small-for-size risk for the donor [5,6]. Furthermore, a recent review has shown that morbidity and mortality of RL procurement still remains a concern, leading to a restriction in the development of LDLT [7].

In such a dilemma of graft selection, the right posterior segment (RPS) graft, or the right lateral sector graft, was introduced as an alternative liver graft to overcome these problems and secure donor safety [8]. The RPS graft has been used as a part of dual grafts [9], however the RPS graft is not considered as a common graft due to technical concerns, surgical difficulties and limited experiences. Indeed, the RPS grafts accounted for only $1.5 \%$ of the LDLT performed in Japan [10]. Although the experience of the RPS graft is limited, the current evidence is of interest in terms of outcomes, strategy and surgical techniques for the RPS graft.

To our best knowledge, the impact of the RPS graft on outcomes has not yet been thoroughly investigated in LDLT. The aim of this study was to review published studies reporting postoperative outcomes of the RPS grafts in LDLT and identify the proposed strategy, anatomical challenges, and surgical techniques for the RPS graft procurement.

\section{Material and methods}

\subsection{Search strategy and data extraction}

The present analysis is reported according to the guidelines of Preferred Reporting Items for Systematic Reviewers and Meta-Analyses (PRISMA) [11]. Major public medical and scientific databases were searched to identify all original articles that report the outcome of the RPS grafts in LDLT.

The search was limited to reports in English language without any limitations regarding the year of publication. The systematic literature search was conducted on the 12th of July 2018 and applied to five databases: Embase, Medline Ovid, Web of Science, Cochrane CENTRAL, and Google scholar. Appropriate queries were conducted with search strings and corresponding terms (Supplementary Table 1).

We included all studies that reported the outcomes of RPS grafts in donors or recipients undergoing LDLT. If the same institution published multiple studies, we selected the most recent article focusing on the outcomes of the RPS grafts. Records without abstracts, conference abstracts, case reports, and reviews were excluded from this analysis.

Duplicate records were removed and two investigators determined eligible papers for further analysis by screening of titles and abstracts independently. Full-text articles were subsequently retrieved and examined independently by two investigators to meet the inclusion criteria and at least one outcome variable. Then, we extracted information on year and country of study publication, study design, donor and recipient information, donor selection, surgical technique, operative outcomes, graft volume and postoperative outcomes.

\subsection{Outcome variables}

The primary outcome was postoperative complications and mortality in donors and recipients. Postoperative minor complications were defined according to the Clavien-Dindo classification (CDc) 1-2, and major complications were defined according to the CDc 3-4 [12]. Secondary outcome included biliary complications in donors and recipients, vascular complications in recipients, and 5-year recipient survival. Furthermore, we reviewed donor selection for the RPS grafts, anatomical challenges of the RPS graft procurement, and surgical technique for the RPS graft procurement.

\section{Results}

\subsection{Characteristics of included studies}

According to our search strategy, we identified 6 studies fulfilling the inclusion criteria (Fig. 1) [13-18]. The characteristics of the eligible articles are shown in Table 1 . All studies were retrospective series from Asian countries ( 2 from Korea and 4 from Japan). A total of 80 donors and recipients with RPS grafts were included in this study. The average donor and recipient age were 38 to 47 years and 47 to 52 years, respectively.

\subsection{Reported outcomes}

\subsubsection{RPS graft donors}

Characteristics of outcomes in donors of the RPS grafts are summarized in Table 2. The average operative time and blood loss were $461 \mathrm{~min}$ (range 418-565) and $557 \mathrm{ml}$ (range 397-889). In addition, the average RPS graft volume was $492 \mathrm{~g}$ (range 437-542). Postoperative reported major and minor complications varied from $4 \%$ to $18 \%$ and from $18 \%$ to $54 \%$, respectively. Accordingly, the overall incidence of major and minor complications was $5.6 \%$ and $34.8 \%$. No mortality after RPS hepatectomy was identified. The overall incidence of bile leakage was $11.1 \%$ after RPS hepatectomy.

\subsubsection{RPS graft recipients}

Characteristics of outcomes in recipients of the RPS grafts are described in Table 3. The average operative time and blood loss were $808 \mathrm{~min}$ (range 662-929) and $5257 \mathrm{ml}$ (range 1430-10192). The average GRWR was 0.837 (0.75-0.90). Only one study [13] reported overall major complications of 54\% (15 events in 28 recipients), while the definition of complications according to the CDc was unclear in other studies. The overall mortality rate for recipients was 14.5\% (10 events in 69 recipients). We found 6 bile leakages (8.7\%), 13 biliary stenosis (18.8\%), 


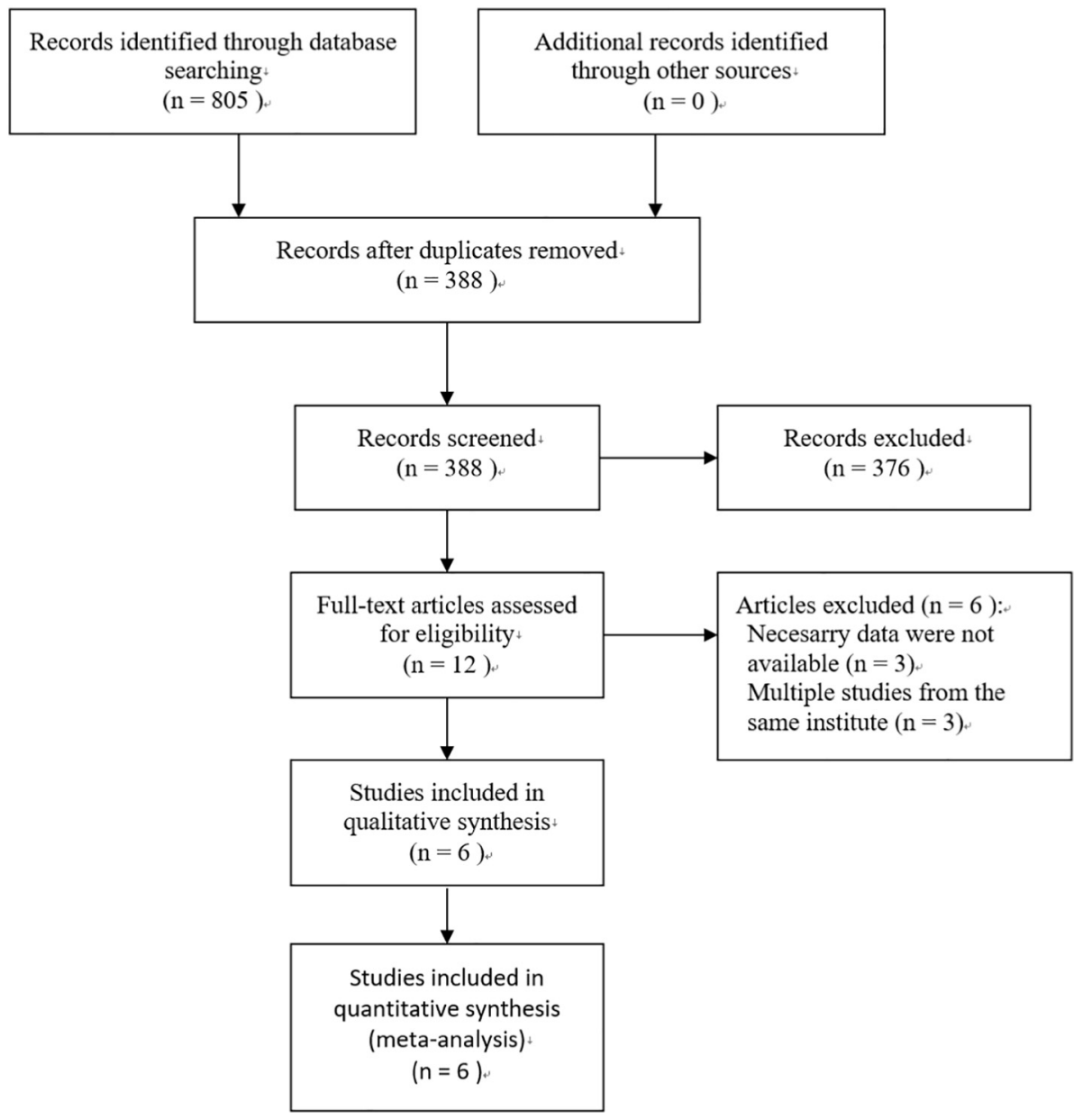

Fig. 1. PRISMA 2009 flow diagram.

6 hepatic artery thrombosis (8.7\%), and 2 re-transplantation (2.9\%) among the 69 recipients. The causes of re-transplantation were due to hepatic artery thrombosis (HAT) and biliary complications accompanied with fibrosing cholestatic hepatitis. Regarding the long-term outcome, one study [15] reported a 3-year patient survival rate of $100 \%$, and three studies $[13,17,18]$ reported the 5 -year patient survival rates of $50 \%$ to $80.8 \%$, however two studies $[14,16]$ did not report it.

\subsubsection{Donor selection for the RPS graft}

The Tokyo group first devised the RPS graft in 2001 to overcome borderline graft-recipient size mismatch [8]. They used the selection criteria based on the recipient's SLV [13]. The RPS graft was considered as the second choice when the left lobe graft did not meet the following criteria: the graft volume was $>40 \%$ of recipient's SLV and 35\% for lowrisk recipients. They considered the branching off of other segments' bile ducts from the posterior HD as a contraindication for the RPS graft procurement. Furthermore, their proposed anatomical contraindications for the RPS graft included the grafts that require separate arterial anastomoses for segment 6 and 7 , and the grafts with supra-portal right posterior hepatic artery (HA).

The Asan group [14] suggested the importance of precise assessment of the RPS volume and portal vein (PV) anatomy. They classified the anatomical variants of PV into three types: type 1 (bifurcation), type 2 (trifurcation), and type 3 (separate RPS branch from the main PV) [19]. When the left lobe volume was $<30 \%$ of whole liver volume and the PV shows type 3 anatomy, they considered the possibility of RPS graft procurement. Furthermore, they concluded livers with type 1 or type 2 PV would not be suitable for RPS graft procurement.

The Ajou group [15] based their selection criteria for living liver donors based on age 18 to 55 years, volumetric criteria and a degree of microvesicular steatosis $<30 \%$. Regarding the volumetric criteria, GRWR had to be $>0.7 \%$ for recipients with low Model for End-Stage Liver Disease (MELD) scores $(<15)$ and $>0.8 \%$ for recipients with high MELD scores $(\geq 15)$. The RPS graft was considered when the left lobe

Table 1

Study characteristics.

\begin{tabular}{|c|c|c|c|c|c|c|c|}
\hline Study & Year & Country & Study design & Number & Donor age & Recipient age & Recipient MELD \\
\hline Kokudo et al. [13] & 2016 & Japan & Retrospective & 28 & $40(30-49)$ & $47(31-53)$ & $15.7(13.3-21.6)$ \\
\hline Hwang et al. [14] & 2004 & Korea & Retrospective & 6 & n.a. & n.a. & n.a. \\
\hline Kim et al. [15] & 2011 & Korea & Retrospective & 13 & $38(18-50)$ & $52(39-65)$ & $13(6-29)$ \\
\hline Marubashi et al. [16] & 2011 & Japan & Retrospective & 11 & $42.2(13.3)$ & n.a. & n.a. \\
\hline Yoshizumi et al. [17] & 2014 & Japan & Retrospective & 8 & $41.5(13.0)$ & $50.9(11.9)$ & $18.4(6.8)$ \\
\hline Hori et al. [18] & 2015 & Japan & Retrospective & 14 & $47.1(10.4)$ & $47.4(12.8)$ & $22.4(12.3)$ \\
\hline
\end{tabular}

Values are presented as means (standard deviation) or medians (interquartile range).

MELD, Model for End-Stage Liver Disease; n.a., not available. 
Table 2

Literatures reporting outcomes in donors of the right posterior segment grafts.

\begin{tabular}{|c|c|c|c|c|c|c|c|c|}
\hline Study & Number & Operative time (min) & Blood loss (ml) & Graft volume (g) & Minor complications & Major complications & Mortality & Bile leakage \\
\hline Kokudo et al. [13] & 28 & $565(525-633)$ & $558(407-868)$ & $483(444-523)$ & $10(36)$ & $1(4)$ & $0(0)$ & $6(21)$ \\
\hline Hwang et al. [14] & 6 & n.a. & n.a. & n.a. & n.a. & $0(0)$ & $0(0)$ & $0(0)$ \\
\hline Kim et al. [15] & 13 & $418(40)$ & $539(148)$ & $542(71)$ & $7(54)$ & $1(8)$ & $0(0)$ & $2(15)$ \\
\hline Marubashi et al. [16] & 11 & $454(57)$ & $889(534)$ & $499(82)$ & $2(18)$ & $2(18)$ & $0(0)$ & $0(0)$ \\
\hline Yoshizumi et al. [17] & 8 & $442(116)$ & $402(211)$ & $437(57)$ & n.a. & n.a. & $0(0)$ & n.a. \\
\hline Hori et al. [18] & 14 & $426(90)$ & $397(274)$ & $499(91)$ & $4(29)$ & $0(0)$ & $0(0)$ & $0(0)$ \\
\hline
\end{tabular}

Values are presented as means (standard deviation), medians (interquartile range) or numbers (percentages).

n.a., not available.

volume of the donor liver was too small ( $<35 \%$ of total liver volume) for the RL procurement and when RPS volume was greater than the left lobe volume. They had no exclusion criteria involving the vascular and biliary anatomy of the liver.

The Osaka group [16] considered the RPS graft when the left lobe and the RL did not fulfill the following criteria: (1) an estimated volume of the liver remnant of $>35 \%$ of donor total liver volume; and (2) an estimated donor graft liver volume of $>40 \%$ of the recipient's standard liver volume (SLV).

The Kyushu group [17] reported that the RPS graft was considered when the graft volume/SLV ratio with the left lobe was $<35 \%$ and the remnant donor liver volume after the RL hepatectomy was $<35 \%$. They also suggested that livers with type $1 \mathrm{PV}$ and the posterior hepatic duct (HD) running through the dorsal side of the posterior PV are not suitable for the RPS grafts hepatectomy.

The Kyoto group [18] indicated the possibility of the RPS graft when GRWR with the left lobe was $<0.6$ and the RPS graft could provide sufficient GRWR while respecting donor safety. Graft selection was primarily determined according to volumetric analyses, then further analysis was conducted by imaging evaluation especially for hepatic vein and biliary duct. In addition, preoperative recognition of territories based on imaging is important to decide the resection line for the RPS graft procurement [20].

\subsubsection{Anatomical challenges of the RPS graft procurement}

The precise understanding of anatomical variations and volumetric analyses plays a critical role in graft selection and surgical strategy for the RPS hepatectomy.

Several branching patterns of the HA have been reported including standard anatomy branching, the replaced left HA from the left gastric artery, and the replaced right HA from the superior mesenteric artery [21-23]. However, special caution for the second-order branches of right HA is required in the RPS hepatectomy. Livers with the secondorder branches of right HA bifurcating within the liver parenchyma or close to the hilar plate might not be suitable for reconstruction because of short stumps [14]. In patients with arterial anomalies such as the anterior HA branched off the posterior HA or very close to the bifurcation of the posterior HA, it is necessary to consider sacrificing the anterior branch to obtain a single orifice of the right posterior HA with less peripheral dissection around the artery for segment 6 and 7 in order to prevent a risk of HAT [13]. Furthermore, the Tokyo group proposed that the presence of a supra-portal right hepatic artery may be a contraindication for the RPS hepatectomy because of the difficulty of the procedure and the high risk of HAT [13].

The branching pattern of the PV is also an important factor for the RPS grafts procurement. Previous studies classified PV variations in three branching patterns (Fig. 2) and reported their prevalence as follows: type 1 (bifurcation), ranging from $65.5 \%$ to $86.4 \%$; type 2 (trifurcation), ranging from $6.3 \%$ to $16.1 \%$; and type 3 (separate posterior PV from the main PV), ranging from $4.2 \%$ to $23.5 \%$ [21-24]. Livers with type 3 PV anatomy would be the most suitable for the RPS grafts procurement in terms of the PV [14,17], otherwise additional surgical technique including venoplasty, dual anastomoses, and graft interpositon would be necessary for PV reconstruction $[13,18]$.

In the literature review, normal biliary anatomy was found in $64.5 \%$ to $80.0 \%$ of all cases, [25] while the presence of the right posterior duct into the left HD was the most common anatomic variant of the biliary system with prevalence between $5.7 \%$ and $19 \%$. Moreover, the presence of the right posterior HD draining into the common HD was reported to be 5.5\% [21]. During the RPS grafts procurement, the extra hepatic division of the right posterior HD could be possible when the right posterior HD drains into the common HD. In other cases, the intrahepatic division of the bile duct is an essential technique for the RPS grafts procurement [15]. From the point of view of the running patterns of the posterior HA, $\mathrm{PV}$, and $\mathrm{HD}$, the posterior HD running through the ventral side of the right PV would be the most suitable for the RPS grafts (Fig. 3A), however the posterior HD running through the dorsal side of the posterior PV would not be favorable (Fig. 3B) [17]. The right posterior HD can be dissected and divided from surrounding connective tissue with pulling the right portal branch cranially [26]. Accordingly, the detailed evaluation for the biliary anatomy is critical to minimize postoperative biliary complications.

Attention to the branching patterns of hepatic vein is mandatory, especially for the middle and inferior right hepatic veins, as insufficient venous outflow could lead to severe congestion with subsequent graft dysfunction and severe complications for the recipient [27]. Therefore, a $V 6$ reconstruction must be considered when the middle hepatic vein drains a significant part of segment VI, which should be confirmed by estimation of the congestion area of segment VI in the RPS graft [28]. The indication for V6 reconstruction is determined not by the diameter

Table 3

Literatures reporting outcomes in recipients with the right posterior segment grafts.

\begin{tabular}{|c|c|c|c|c|c|c|c|c|c|c|c|}
\hline Study & Number & Operative time & Blood loss (ml) & GRWR & $\begin{array}{l}\text { Major } \\
\text { complications }\end{array}$ & Mortality & $\begin{array}{l}\text { Bile } \\
\text { leakage }\end{array}$ & $\begin{array}{l}\text { Biliary } \\
\text { stenosis }\end{array}$ & HAT & Re-LTX & $\begin{array}{l}\text { 5-year survival } \\
\text { (\%) }\end{array}$ \\
\hline Kokudo et al. [13] & 28 & $929(843-984)$ & 4150 (2663-6998) & $0.85(0.74-1.01)$ & $15(54)$ & $2(7)$ & $4(14)$ & $8(29)$ & $4(14)$ & $1(4)$ & 80.8 \\
\hline Hwang et al. [14] & 6 & n.a. & n.a. & n.a. & n.a. & $0(0)$ & $0(0)$ & $1(17)$ & $0(0)$ & $0(0)$ & n.a. \\
\hline Kim et al. [15] & 13 & $662(91)$ & $1430(1384)$ & $0.83(0.19)$ & n.a. & $0(0)$ & $0(0)$ & $0(0)$ & $0(0)$ & $0(0)$ & $100^{\mathrm{a}}$ \\
\hline Marubashi et al. [16] & 11 & n.a. & n.a. & $0.86(0.18)$ & n.a. & n.a. & n.a. & n.a. & n.a. & n.a. & n.a. \\
\hline Yoshizumi et al. [17] & 8 & n.a. & n.a. & $0.75(0.16)$ & n.a. & $2(25)$ & $1(13)$ & $4(50)$ & $0(0)$ & $1(13)$ & 75 \\
\hline Hori et al. [18] & 14 & $834(180)$ & $10,192(10703)$ & $0.897(0.203)$ & n.a. & $6(43)$ & $1(7)$ & $1(7)$ & $2(14)$ & $0(0)$ & 50 \\
\hline
\end{tabular}

Values are presented as means (standard deviation), medians (interquartile range) or numbers (percentages).

GRWR, graft-to-recipient weight ratio; HAT, hepatic artery thrombosis; Re-LTX, liver retransplantation; n.a., not available.

a 3 -year patient survival rate. 
A

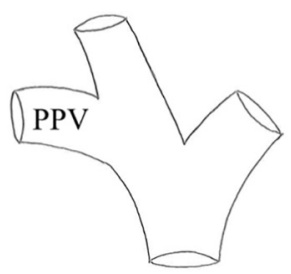

B

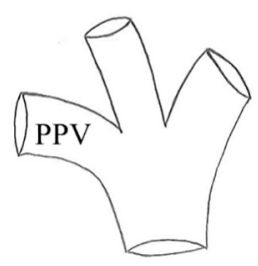

$\mathrm{C}$

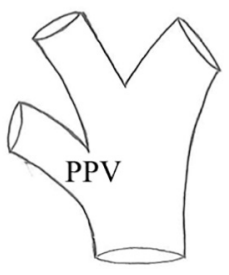

Fig. 2. Classification of portal vein systems. A, Type 1 (bifurcation). B, Type 2 (trifurcation). $\mathrm{C}$, Type 3 (separate posterior portal vein from the main portal vein).

of V6, but by the volume of the congestion area [28]. An inferior RHV is found in approximately $40 \%$ to $60 \%$ of all donors [22,29], and a major inferior RHV, larger than $5 \mathrm{~mm}$, is an indication for reconstruction [5].

A previous study regarding volumetric analysis showed that $25 \%$ of donors had a large RL measuring $>70 \%$ of the whole liver volume [30]. Among these donors, $72 \%$ had a larger RPS than left lobe with caudate lobe, which means $18 \%$ of donors could be a candidate for the RPS graft procurement. In the cases where RL procurement would produce an insufficient donor liver remnant [5], the RPS graft procurement could be an alternative graft option respecting donor's safety in regards to the liver remnant size.

\subsubsection{Surgical technique for the RPS graft procurement}

The Tokyo group [13] reported that after extra hepatic hilar dissection to isolate the right posterior HA and posterior PV, hepatectomy was performed with inflow occlusion. During transection of the liver parenchyma, the posterior HD is divided based on the findings of intraoperative cholangiography. In addition, they recommended to consider the V6 reconstruction when hepatic venous congestion in the RPS graft was confirmed after parenchymal transection [28].

The Asan group [14] confirmed the demarcation line first by clamping the posterior PV and the posterior HA, then the RPS graft was procured only when it appeared definitely larger than the left lobe. The procurement of the RPS graft was similar to that of the RL graft. Regarding the bile duct division, the radio-opaque marker tagging method was used to identify an appropriate site of bile duct division.

The Ajou group [15] performed the RPS procurement similarly to the RL hepatectomy. After full mobilization of the RL and hilar dissection, the parenchymal dissection was performed along the demarcation line. After parenchymal division, the intrahepatic Glissonian sheath of the right posterior portal pedicle was exposed, a radiopaque marker was placed on the sheath, and intraoperative cholangiography was performed to decide the cutting line of posterior HD. Then, the posterior $\mathrm{HD}$, the posterior HA, the posterior PV, and the right hepatic vein (RHV) were divided.

A

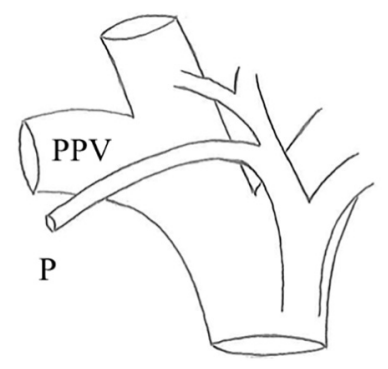

B

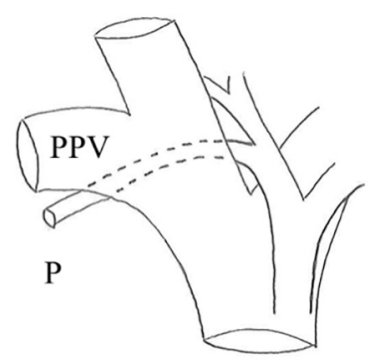

Fig. 3. Running patterns of the posterior hepatic duct and portal vein systems. A, The posterior hepatic duct (HD) running through the ventral side of the right portal vein (PV) would be the most suitable for the right posterior segment (RPS) grafts. B, The posterior HD running through the dorsal side of the posterior PV would not be favorable.

The Kyushu group [17] presented two retrieval techniques including the conventional RPS graft retrieval and the extended RPS graft retrieval. For the conventional RPS graft retrieval, the posterior Glissonian pedicle was clamped, and the objective area was harvested as the RPS graft. Parenchymal transection was performed toward the root of the posterior Glissonian pedicle and the RHV. For the extended RPS graft retrieval, the drainage area of the RHV was procured. The anterior fissure was opened, and the transection line was directed straight to the root of the RHV. The division line of posterior HD was determined by intraoperative cholangiography.

The Kyoto group $[18,20]$ suggested that the cut line for the RPS graft should be carefully considered based on the demarcation line and the RHV (Fig. 4A). However it is necessary to keep in mind that segmental territory based on inflow and that based on outflow never overlap completely in the RPS graft. They preserved the middle hepatic vein territory from segment 5 and 8 in the remnant liver for the donor safety. Posterior branches of the HA and PV were dissected (Fig. 4B). Liver resection was performed with a hanging maneuver technique, and the RPS graft was procured after the transection of biliary duct, HA, and PV for the RPS (Fig. 4C).

\section{Discussion}

This systematic review demonstrated clinical outcomes of the RPS graft for donors and recipients in LDLT. Several donor selection criteria, anatomy and surgical techniques for the RPS graft procurement have been reported from Asian countries. The overall incidences of major and minor complications after the RPS graft procurement were 5.6\% and $34.6 \%$, respectively. In addition, no study reported donor death after the RPS procurement. Recipients of the RPS grafts had the following risks of postoperative complications: overall mortality rate of $14.5 \%$, bile leakage of $8.7 \%$, biliary stenosis of $18.8 \%$, hepatic artery thrombosis of $8.7 \%$, and liver re-transplantation of $2.9 \%$. A recent review regarding RL procurement has reported 23 donor deaths between 1999 and 2017 , and high morbidity ranging from $10 \%$ to $78.3 \%$ [7]. Regarding recipients' outcome, two of the included studies reported outcome in the RPS graft and the RL graft [13,15]. Kim et al. [15] demonstrated no significant differences between the groups in postoperative mortality, vascular complications, biliary complications, and 3-year survival [15]. Kokudo et al. [13] reported that the RPS graft recipients had significantly higher incidence of major complications $(P<.001)$ and HAT $(P=.04)$ even though other complications were not significant. They concluded that peripheral dissection of the artery for segment 6 and 7 was associated with a high risk of HAT.

Accordingly, the postoperative outcomes of the RPS graft for donors and recipients were acceptable when respecting careful donor selection and precise anatomical evaluation. However, it should be noted that the numbers of RPS grafts in LDLT are very limited and this procedure is by far predominantly performed in experienced high volume liver transplant centers in Asian countries. With the requisite experience, the RPS graft could be an alternative if there is no deceased donor available and living donors show graft-volume mismatch for right or left lobe donation.

The present study has several limitations. First, the included studies' sample sizes were small, were retrospective and exclusively from Asian countries with different follow-up period. Therefore there might be a potential selection bias regarding the indication for LDLT with RPS grafts. Second, the prognosis in recipients with the RPS grafts was controversial because of limits in data reporting. Third, the included cases took place over a long time. Therefore, information about numbers of surgeons, their individual experience and their learning curve were lacking or not described in all studies. Finally, well-designed studies comparing the significance of the RPS grafts, RL grafts and LL grafts would be very difficult. There is also no room to randomize the usage of different grafts in a study. 

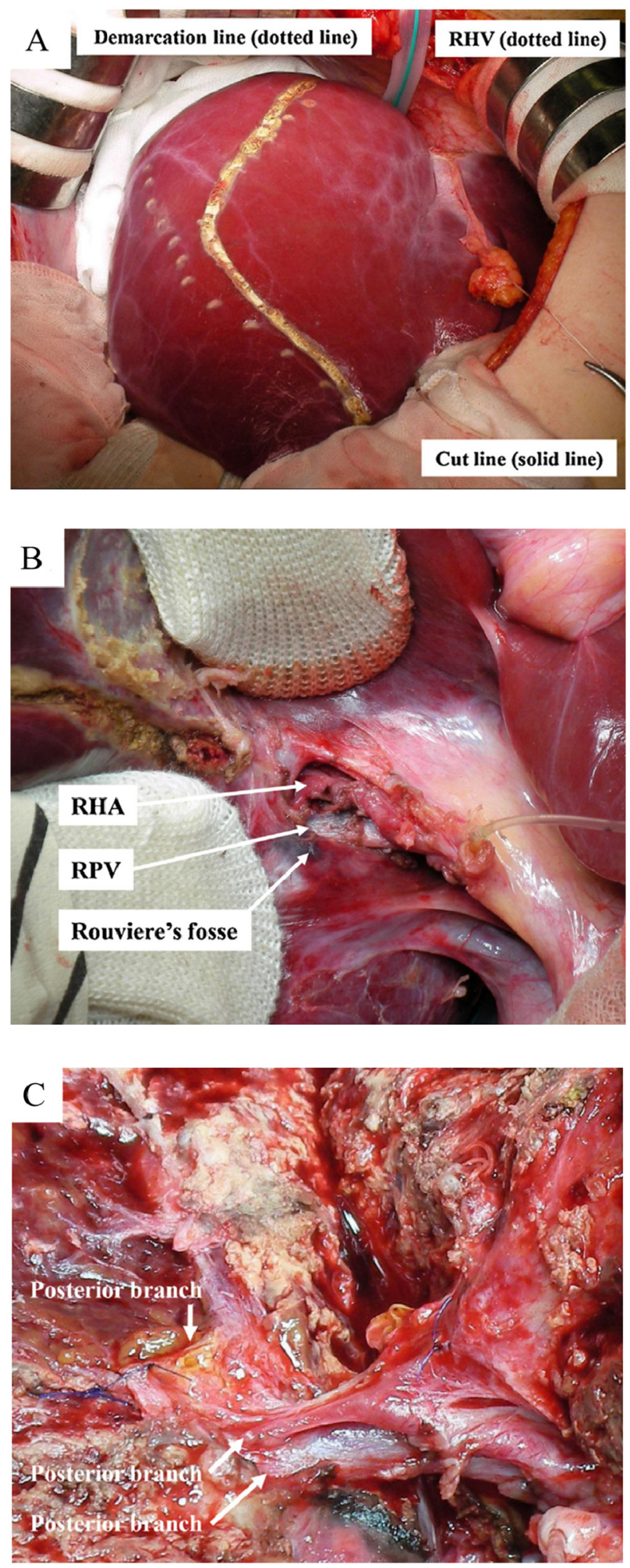

Fig. 4. The right posterior segment graft procurement. A, The right hepatic vein and the demarcation line were marked with dotted line, and the cut line was carefully decided with solid line based on them. B, Posterior branches of the hepatic artery and portal vein were dissected. C, Liver resection was performed with a hanging maneuver technique, and the RPS graft was procured after the transection of biliary duct, hepatic artery, and portal vein for the right posterior segment. These pictures are cited. [20]

\section{Conclusions}

The present study suggests that the RPS graft can be considered as an alternative liver graft in exceptional circumstances. This procedure can be performed safely with strict donor selection and optimal surgical strategy. The precise evaluation and understanding of anatomical variations including HA, PV, HD, and hepatic vein is critical for selecting donors and planning the surgical strategy in the RPS grafts procurement. Last but not least, the RPS graft LDLT should only be done in centers with a high level of experience in LDLT. As the experience in this procedure is limited to a few centers in Asian countries, it will be difficult to gain the required expertise in the future. It would be impractical to consider a trial to randomize LDLT patients based on the lobar and sectoral nature of the graft.

\section{Funding}

The authors declare that they received no funding for this study.

\section{Declaration of Competing Interest}

The authors declare no conflicts of interest.

\section{Acknowledgements}

We express our gratitude to Wichor M. Bramer (Biomedical Information Specialist) from the Medical Library in Erasmus MC, Erasmus University Medical Centre Rotterdam (Rotterdam, the Netherlands) for his involvement in constructing research queries in databases.

\section{Appendix A. Supplementary data}

Supplementary data to this article can be found online at https://doi. org/10.1016/j.trre.2019.100510.

\section{References}

[1] Abt PL, Mange KC, Olthoff KM, Markmann JF, Reddy KR, Shaked A. Allograft survival following adult-to-adult living donor liver transplantation. Am J Transplant 2004;4: $1302-7$.

[2] Thuluvath PJ, Yoo HY. Graft and patient survival after adult live donor liver transplantation compared to a matched cohort who received a deceased donor transplantation. Liver Transpl 2004;10:1263-8.

[3] Lo CM. Deceased donation in Asia: challenges and opportunities. Liver Transpl 2012; 18:S5-7.

[4] Quintini C, Hashimoto K, Uso TD, Miller C. Is there an advantage of living over deceased donation in liver transplantation? Transpl Int 2013;26:11-9.

[5] Lee SG. A complete treatment of adult living donor liver transplantation: a review of surgical technique and current challenges to expand indication of patients. Am J Transplant 2015;15:17-38.

[6] Chen CL. The right posterior sector graft in living donor liver transplantation revisited. Liver Transpl 2014;20:1019-20.

[7] Brige P, Hery G, Chopinet S, Palen A, Azoulay D, Gregoire E. Morbidity and mortality of hepatic right lobe living donors: systematic review and perspectives. J Gastrointestin Liver Dis 2018;27:169-78.

[8] Sugawara Y, Makuuchi M, Takayama T, Mizuta K, Kawarasaki H, Imamura H, et al. Liver transplantation using a right lateral sector graft from a living donor to her granddaughter. Hepatogastroenterology 2001;48:261-3.

[9] Hwang S, Lee SG, Lee YJ, Sung KB, Park KM, Kim KH, et al. Lessons learned from 1,000 living donor liver transplantations in a single center: how to make living donations safe. Liver Transpl 2006;12:920-7.

[10] Umeshita K, Inomata Y, Furukawa H, Kasahara M, Kawasaki S, Kobayashi E, et al. Liver transplantation in Japan: registry by the Japanese Liver Transplantation Society. Hepatol Res 2016;46:1171-86.

[11] Moher D, Liberati A, Tetzlaff J, Altman DG, PRISMA Group. Preferred reporting items for systematic reviews and meta-analyses: the PRISMA statement. BMJ 2009;339: b2535.

[12] Clavien PA, Barkun J, de Oliveira ML, Vauthey JN, Dindo D, Schulick RD, et al. The Clavien-Dindo classification of surgical complications: five-year experience. Ann Surg 2009;250:187-96.

[13] Kokudo T, Hasegawa K, Arita J, Yamamoto S, Kaneko J, Akamatsu N, et al. Use of a right lateral sector graft in living donor liver transplantation is feasible, but special caution is needed with respect to liver anatomy. Am J Transplant 2016;16:1258-65.

[14] Hwang S, Lee SG, Lee YJ, Park KM, Kim KH, Ahn CS, et al. Donor selection for procurement of right posterior segment graft in living donor liver transplantation. Liver Transpl 2004;10:1150-5.

[15] Kim BW, Xu W, Wang HJ, Park YK, Lee K, Kim MW. Volumetry-based selection of right posterior sector grafts for adult living donor liver transplantation. Liver Transpl 2011;17:1046-58. 
[16] Marubashi S, Nagano H, Wada H, Kobayashi S, Eguchi H, Takeda Y, et al. Donor hepatectomy for living donor liver transplantation: learning steps and surgical outcome. Dig Dis Sci 2011;56:2482-90.

[17] Yoshizumi T, Ikegami T, Kimura K, Uchiyama H, Ikeda T, Shirabe K, et al. Selection of a right posterior sector graft for living donor liver transplantation. Liver Transpl 2014;20:1089-96.

[18] Hori T, Kirino I, Uemoto S. Right posterior segment graft in living donor liver transplantation. Hepatol Res 2015;45:1076-82.

[19] Lee SG, Hwang S, Kim KH, Ahn CS, Park KM, Lee YJ, et al. Approach to anatomic variations of the graft portal vein in right lobe living-donor liver transplantation. Transplantation 2003;75:S28-32.

[20] Hori T, Oike F, Ogura Y, Ogawa K, Uemoto S. Graft harvest of right posterior segment for living-donor liver transplantation. Int J Surg Case Rep 2014;5:516-22.

[21] Varotti G, Gondolesi GE, Goldman J, Wayne M, Florman SS, Schwartz ME, et al. Anatomic variations in right liver living donors. J Am Coll Surg 2004;198:577-82.

[22] Uchida K, Taniguchi M, Shimamura T, Suzuki T, Yamashita K, Ota M, et al. Threedimensional computed tomography scan analysis of hepatic vasculatures in the donor liver for living donor liver transplantation. Liver Transpl 2010;16: 1062-8.
[23] Okten RS, Kucukay F, Dedeoglu H, Akdogan M, Kacar S, Bostanci B, et al. Branching patterns of the main portal vein: effect on estimated remnant liver volume in preoperative evaluation of donors for liver transplantation. Eur J Radiol 2012;81:478-83.

[24] Atasoy C, Ozyürek E. Prevalence and types of main and right portal vein branching variations on MDCT. AJR Am J Roentgenol 2006;187:676-81.

[25] Uysal F, Obuz F, Uçar A, Seçil M, Igci E, Dicle O. Anatomic variations of the intrahepatic bile ducts: analysis of magnetic resonance cholangiopancreatography in 1011 consecutive patients. Digestion 2014;89:194-200.

[26] Sugawara Y, Matsui Y, Noritomi T, Kaneko J, Makuuchi M. Safe bile duct division in right lateral sector graft. Hepatogastroenterology 2005;52:170-2.

[27] Lee S, Park K, Hwang S, Lee Y, Choi D, Kim K, et al. Congestion of right liver graft in living donor liver transplantation. Transplantation 2001;71:812-4.

[28] Hashimoto T, Sugawara Y, Kishi Y, Akamatsu N, Matsui Y, Kokudo N, et al. Reconstruction of the middle hepatic vein tributary in a right lateral sector graft. Liver Transpl 2005;11:309-13.

[29] Nakamura S, Tsuzuki T. Surgical anatomy of the hepatic veins and the inferior vena cava. Surg Gynecol Obstet 1981;152:43-50.

[30] Leelaudomlipi S, Sugawara Y, Kaneko J, Matsui Y, Ohkubo T, Makuuchi M. Volumetric analysis of liver segments in 155 living donors. Liver Transpl 2002;8:612-4. 